\title{
NS-PROVENIENZFORSCHUNG AN DER MEDIZINISCHEN UNI- VERSITÄT WIEN 2013 UND 2014. RESTITUTION VON BÜCHERN AUS DEM ANTIQUARIAT HANS PETER KRAUS SOWIE DER VER- LAGSBUCHHANDLUNG ANZENGRUBER-VERLAG, BRÜDER SU- SCHITZKY
}

von Bruno Bauer und Walter Mentzel

Zusammenfassung: 2014 wurden von der Medizinischen Universität Wien in zwei Fällen von NS-Raubgut Bücher an die rechtmäßigen Eigentümer bzw. deren Erben restituiert. Die Restitutionen betrafen das „Antiquariat Hans Peter Kraus“ und „Verlagsbuchhandlung Anzengruber-Verlag, Brüder Suschitzky“.

Schlüsselwörter: Medizinische Universitätsbibliothek; NS-Provenienzforschung; Bücherraub; Restitution; Antiquariat Hans Peter Kraus; Verlagsbuchhandlung Anzengruber Verlag - Brüder Suschitzky

NS-PROVENANCE-RESEARCH-PROJECT OF THE MEDICAL UNIVERSITY OF VIENNA IN 2013 AND 2014: RESTITUTION OF BOOKS FROM „ANTIQUARIAT HANS PETER KRAUS“ AND „VERLAGSBUCHHANLDUNG ANZENGRUBER-VERLAG, BRÜDER SUSCHITZKY“

Abstract: In 2014 in two cases the Medical University of Vienna was able to elicit the rightful owners/heirs of expropriated goods during the Nazi-regime. Restitution was made to the heirs of Hans Peter Kraus and Philipp and Wilhelm Suschitzky.

Keywords: Medical University of Vienna; university library; NS-provenance-research; looted books; „Antiquariat Hans Peter Kraus“; „Verlagsbuchhandlung AnzengruberVerlag; Brüder Suschitzky" 


\section{Einleitung}

Seit 2007 wird an der Universitätsbibliothek der Medizinischen Universität Wien systematisch NS-Provenienzforschung betrieben. Seit 2010 erfolgen laufend Restituierungen jener Bücher, die von der Universitätsbibliothek bzw. ihren Vorgängerinstitutionen unrechtmäßig erworben worden sind. 2010 wurden 39 Bücher aus dem Besitz von Carl Julis Rothberger (18711945) an dessen Tochter Bertha Gutmann restituiert ${ }^{1}, 2011$ erfolgte die Restitution eines Buches an die Friedrich-Ebert-Stiftung in Deutschland, 2012 wurden fünf Bücher aus der Privatbibliothek des Raoul Fernand Jellinek-Mercedes (1888-1939) an die rechtmäßigen Erben und ein Buch aus der Privatbibliothek Alfred Arnstein (1886-1972) an dessen in England lebenden Sohn restituiert. ${ }^{2} 2014$ wurden, basierend auf intensiven Recherchen, weitere unrechtmäßig erworbene Bücher restituiert.

Die beiden Restitutionsfälle, Antiuariat „Hans Peter Kraus“ bzw. Verlagsbuchhandlung „Anzengruber-Verlag, Brüder Suschitzky“, die 2014 durch Restitution abgeschlossen werden konnten, gehören zu jenen Beispielen, die dokumentieren, in welchen Ausmaß und in welchen Formen Antiquariate von der Judenverfolgung profitierten.

\section{Antiquariat Hans Peter Kraus}

Im Mai 2014 wurden 16 Bücher an eine in New York lebende Tochter von Hans Peter Kraus, stellvertretend für die noch lebenden erbberechtigten Kinder von Hans Peter Kraus, restituiert. Es handelt sich dabei um zwölf Titel, die an der Zweigbibliothek für Geschichte der Medizin (vormals Institut für Geschichte der Medizin) per Autopsie am Bücherregal aufgefunden wurden.

1) Stoll Otto, Suggestion und Hypnotismus in der Völkerpsychologie. 2. Aufl. Verlag von Veit \& Comp.: Leipzig 1904. (Inv.-Nr. 10.886)

2) Wallich Emanuel Wolfgang, Anleitung für Mütter zur Ernährung und Behandlung der Kinder in den ersten zwey Lebensjahren. Rößl und Kaulfuß: Wien 1810. (Inv.-Nr. JB 4.089)

3) Squirrel R, An essay on indigestion and its consequences, or advice to persons afflicted with debility of the digestive organs, nervous and bilious disorders, the gout, etc. With the Method of curing the above complaints; wherein are observations on animal and vegetable food, together with Milk, Drinks, Exercise, and Air: Also remarks on sea or cold bathing, Distinguishing the different States of Consti- 
tution, in which bathing will be found salutary or pernicious: Likewise explaining the reason Why the sea air is more salubrious than that of Cities and Inland Places. 2nd Edition. Murray and Highley: London [1799]. (Inv.-Nr. JB 2.361)

4) Besse [Jean de], Lettre a Moniseur [Jean Adrien Helvetius] auteur du nouveau livre de l'oeconomie animale, \& des Observations sur les petites veroles. François Fournier: Paris 1723. (Inv.-Nr. JB 4.886)

5) Zimmermann W.F.A, Magnetismus und Mesmerismus oder Physische und geistige Kräfte der Natur. Der mineralische und thierische Magnetismus sowohl in seiner wirklichen Heilkraft, als in dem Mißbrauch, der von Betrügern und Narren damit getrieben worden, im Zusammenhange mit der Geisterklopferei - der Tischrückerei - dem Spiritualismus. 2. Aufl. Verlag von Theodor Thiele: Berlin 1863. (Inv.-Nr. 13.858)

6) Lederer $\operatorname{Fr}[\mathrm{anz}] \mathrm{Xav}[\mathrm{er}]$ von, Über die wesentlichen Vortheile der Einimpfung der Kinderblattern. Für das Landvolk geschrieben. Franz Joseph Rößel: Wien 1797. (Inv.-Nr. JB 6.657)

7) Descartes René, Les principes de la philosophie. 4. Aufl. Theodore Girard: Paris 1681. (Inv.-Nr. JB 5.596)

8) Fauken J[ohann] P[eter] [Franz] Xaver, Das in Wien im Jahre 1771 und 1772 sehr viele Menschen anfallende Fäulungsfieber. Samt Anhang einer bösartigen Krankheit, welche im Jahre 1770 unter den Kindbetterinnen im Spitale zu St. Marx gewütet hat. Joh. Thom. Edlen v. Trattnern: Wien 1772. (Inv.-Nr. JB 5.081)

9) Ozanam [ean] $A[$ ntoine] $F[$ rançois]: Histoire médicale générale et particulière des maladies pidémiues, contagieuses et épizootiques. Qui ont régné en Europe depuis les temps les plus reculés; et notamment depuis le XIVe siècle jusqu'à nos jours. Tome 1-5. MéquignonMarvis: Paris et Lyon 1817-23. (Inv.-Nr. JB 5.138/1-5)

10) Godfrey $C$ [harles] $B$ [oyle]: An historical and practical treatise on the venereal disease; dedicated to His Grace The Duke Of Queensbury. Illustrated with some remarkable cases; being the result of fifteen years extensive practice in this metropolis: Together with observations on a late publication of Dr. Buchan's, on this complaint: In which his Principles are candidly examined, and clearly refuted. In this work is laid down a mode of prevention, which, if universally adopted, will, in a few years, annihilate this inveterate disease. H. D. Symonds: London (1797) 1800. (Inv.-Nr. JB 4.673)

11) Wundt Wilhelm: Erlebtes und Erkanntes. 2. Aufl. Alfred Körner Verlag: Stuttgart 1921. (Inv.-Nr. 10.709) 
12) Swoboda Hermann: Die Perioden des menschlichen Organismus in ihrer psychologischen und biologischen Bedeutung. Franz Deutikke: Leipzig und Wien 1904. (Inv.-Nr. 10.890)

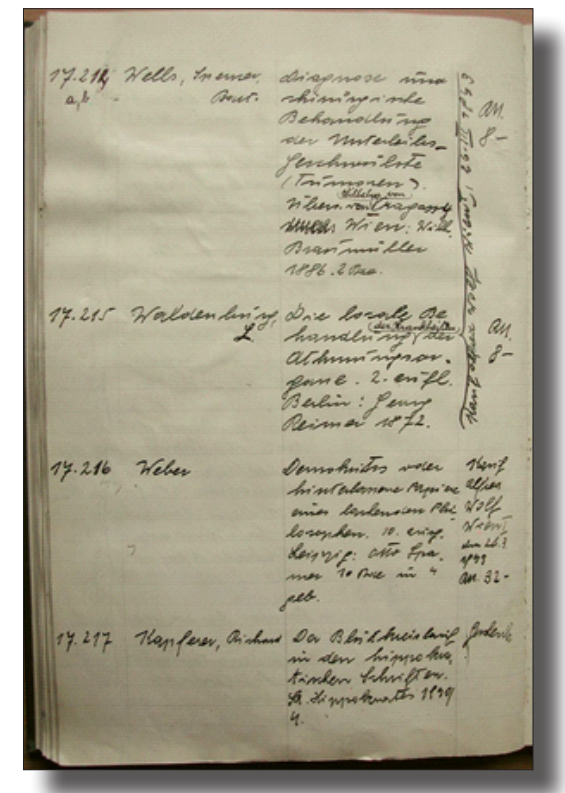

Abb. 1: Beispiel einer Seite aus dem Inventarbuch der Zweigbibliothek für Geschichte der Medizin. Als Eintragung in der rechten Spalte finden sich die jeweiligen Bezugsquellen: Eintrag 17.216 betrifft den Ankauf über das Antiquariat Wolf

Aufgrund des Inventarverzeichnisses der Zweigbibliothek für Geschichte der Medizin bzw. handschriftlichen Eintragungen in den einzelnen Büchern lässt sich rekonstruieren, dass neun der zwölf betroffenen Titel in den Jahren 1940 bis 1949 durch das Institut für Geschichte der Medizin vom Antiquariat Alfred Wolf angekauft wurden. Folgende Ankaufsdatums scheinen auf: 12.02.1940 (Nr. 7), 29.08.1940 (Nr. 5, Nr. 8), 12.05.1940 (Nr. 11), 17.10.1940 (Nr. 1), 25.03.1941 (Nr. 9), 29.08.1941 (Nr. 10), 23.03.1949 (Nr. 2) und 19.11.1949 (Nr. 3).

In fünf Büchern (Nr. 2, 3, 4, 7 und 10) befindet sich ein Besitzstempel der Thun-Hohenstein'schen Bibliothek („Tetschner Bibliothek“). Hans Peter Kraus hatte diese Bücher 1934 in Prag erworben. Die „Tetschner Bibliothek" war 1933 vom tschechoslowakischen Militär im Zuge der Beschlagnahmung und Einrichtung des Schlosses als Militärquartier nach Prag transferiert und an Antiquariate veräußert worden. 


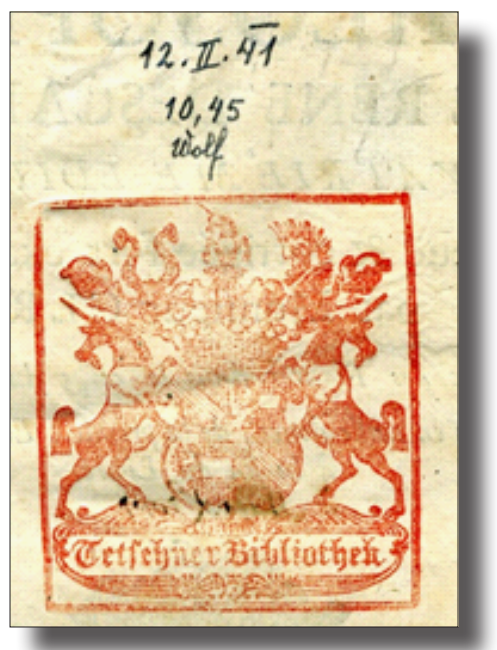

Abb. 2: Besitzstempel der „Tetschner Bibliothek“, darüber Eintrag durch das damalige Institut für Geschichte der Medizin mit dem Kaufvermerk (12.02.1941, RM 10,45, Wolf)

Sieben Titel (Nr. 1, 4, 6, 8, 9, 11 und 12) konnten den von Hans Peter Kraus in den Jahren 1934 und 1937 herausgegebenen Antiquariatskatalogen zugeordnet werden.

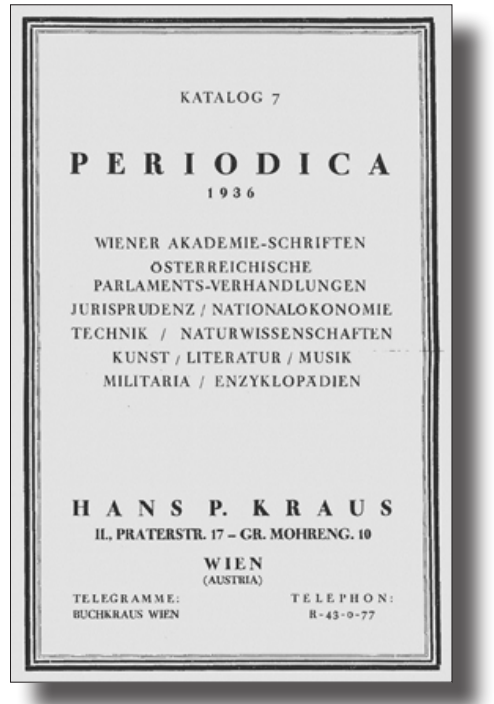

Abb. 3: Katalog 7 Periodica 1936 als Beispiel eines von Hans Peter Kraus in Wien herausgegebenen Antiquariatskataloges 
Hans Peter Kraus, geboren am 12. Oktober 1907 in Wien als Sohn von Emil Kraus, einem Mitglied der Medizinischen Fakultät der Universität Wien, und Hilde Kraus, absolvierte eine Buchhändlerlehre in der Universitätsbuchhandlung R. Lechner in Wien. Danach war er für den Berliner Verlag Wasmuth als Vertreter in Rumänien und Polen und seit 1929 als Mitarbeiter der Leipziger Buchhandlung Karl W. Hiersemann, die eine große und bedeutende antiquarische Abteilung im deutschsprachigen Raum besaß, tätig. 1932 machte er sich selbständig und eröffnete in der Praterstraße im zweiten Wiener Gemeindebezirk eine Antiquariatsbuchhandlung, die in ihrer Blütezeit ca. 100.000 Bände umfasste.

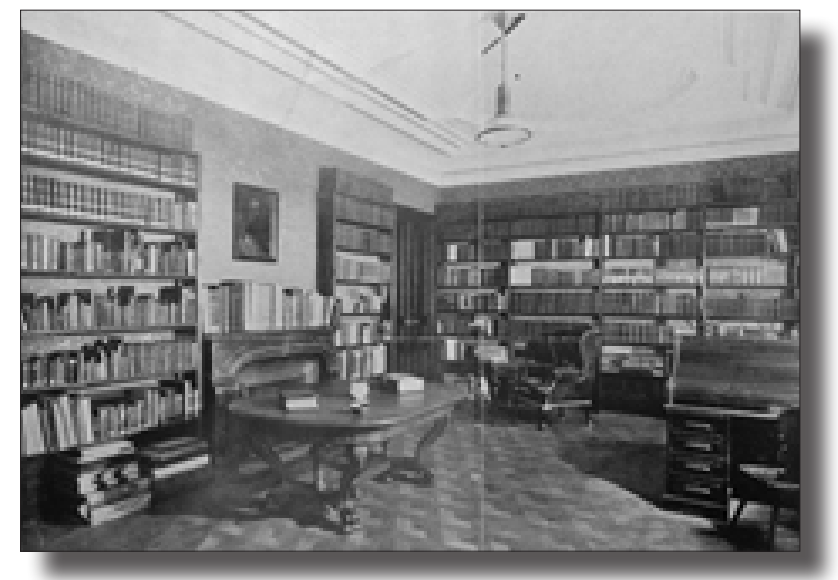

Abb. 4: Innenansicht der in der Praterstraße gelegenen Antiquariatsbuchhandlung „Hans Peter Kraus", um 1930

Nach dem „Anschluss“ im März 1938 wurde er von seinem im Antiquariat beschäftigen Mitarbeiter Alfred Wolf bei der Gestapo angezeigt und infolgedessen in das Konzentrationslager Dachau und danach nach Buchenwald verschleppt. Nach seiner Freilassung gelang Kraus wenige Tage vor Kriegsausbruch die Flucht nach Schweden, von wo er im Oktober 1939 in die USA ausreiste. Nach seiner Ankunft in New York eröffnete er erneut eine Antiquariatsbuchhandlung. In den 1960er Jahren etablierte sich Kraus als einer der renommiertesten Antiquare in den USA. Nach seinem Tod im am 1. November 1988 in Ridgefield (Fairfield County, Connecticut) führte sein Ehefrau Hanni Kraus (geborene Zucker), diese, ebenfalls eine Emigrantin, hatte er 1940 geheiratet, die Geschäfte des Antiquariates noch weitere 15 Jahre. Nach dem Tod von Hanni Kraus im Jahr 2003 beschlossen die als Erben eingesetzten Kinder von Hans und Hanni Kraus, 
das an der 16 East 46th Street in New York liegende Antiquariat zu verkaufen und boten es dem Auktionshaus Sotheby's an.

Am 27. September 1938 gab Hilde Kraus, die Mutter von Hans Peter Kraus, die Vermögensanmeldung beim Ministerium für Arbeit und Wirtschaft, Vermögensverkehrsstelle, erstmals ab, weil sich ihr Sohn zu dieser Zeit im Konzentrationslager Dachau befand. Ebenfalls in seiner Abwesenheit wurde eine zweite Vermögensverkehrsanmeldung am 7. August 1939 durchgeführt. Nach Angaben des bevollmächtigten Rechtsanwalts der Familie Kraus, Dr. Oskar Feigl, wurde eine von der Vermögensverkehrsstelle festgestellte Steuerschuld von Hilde Kraus beglichen, indem sie „den größten Teil ihres Vermögens verpfändet" hat. ${ }^{3}$

Die bisher als geraubt identifizierten Bücher aus der Provenienz des Antiquariates Hans Peter Kraus hat das Institut für Geschichte der Medizin zwischen 1940 und 1949 vom Antiquariat Alfred Wolf angekauft. Wolf war nicht nur für die Inhaftierung seines früheren Arbeitgebers Hans Peter Kraus unmittelbar verantwortlich, er eignete sich auch den gesamten Warenlagerbestand der Firma Hans Peter Kraus an und brachte diesen in das von ihm 1939 neugegründete Antiquariat „Alfred Wolf“ ein. Innerhalb kurzer Zeit wuchs der Bücherbestand durch die „Arisierungen“ eines zweiten Antiquariats (Leo Weiser), durch den Erwerb von Bücherbeständen von „liquidierten“ Antiquariaten oder durch die Übernahme von Privatbibliotheken, die aus dem Zwangsverkauf geflüchteter Juden und Jüdinnen stammten, auf bis zu 600.000 Bücher. ${ }^{4}$ Dieses Antiquariat zählte zwischen 1940 und 1941 zu den Hauptlieferanten der Bibliothek des Instituts für Geschichte der Medizin.

Die Rolle des Antiquariates „Alfred Wolf“ im NS-Bücherraub wurde über die Provenienzforschung hinausgehend durch den Projektleiter Walter Mentzel im Rahmen eines eigenen Projektes zum Thema „NS-Antiquariate und ihre Rolle im nationalsozialistischen Bücherraub unter besonderer Berücksichtigung Wiener Antiquariatsunternehmen "5 detailliert untersucht und die Ergebnisse im Rahmen des 4. Hannoverschen Symposiums 2011 vorgestellt und publiziert. ${ }^{6}$ Damit konnten auch für die Provenienzforschung an anderen Bibliotheken im deutschsprachigen Raum wertvolle Hinweise erarbeitet werden.

Sehr persönliche Eindrücke in die Tätigkeit eines Antiquars liefert Hans Peter Kraus in seiner Selbstbiografie, die 1978 in englischer ${ }^{7}$ und 1982 deutscher Sprache ${ }^{8}$ erschienen ist. Anlässlich seines Todes veröffentlichte die „New York Times“ am 2. November 1988 einen Nachruf. ${ }^{9}$ Auch von seiner Familie wurde eine Gedenkschrift herausgegeben. ${ }^{10}$

Hans Peter und Hanni Kraus haben sich dadurch ein bleibendes Andenken gesetzt, dass sie 1970 eine wertvolle Sammlung über Geschichte und 
Kultur Lateinamerikas während der Kolonialperiode von 1492 bis 1819 der Library of Congress vermacht haben ${ }^{11}$.

\section{Verlagsbuchhandlung „Anzengruber-Verlag, Brüder Suschitzky“}

Im Mai 2014 erfolgte die Restitution eines Buches aus der „Verlagsbuchhandlung der Brüder Philipp und Wilhelm Suschitzky“ an Wolf Suschitzky, den Neffen von Philipp und Sohn von Wilhelm.

Das Buch Börner Wilhelm, Friedrich Jodl, Stuttgart-Berlin, 1911 (Inv.Nr. 17.056) wurde an der Zweigbibliothek für Geschichte der Medizin (vormals Institut für Geschichte der Medizin) per Autopsie am Buchregal als Raubgut identifiziert. Es trägt den handschriftlichen Vermerk „Feb.-Marz 1943“, „1.20“, „Stark“ - ein Hinweis darauf, dass das Buch im Februar/ März 1943 vom Antiquariat Karl Stark um 1.20 Reichsmark erworben wurde. Dieselbe Information befindet sich im Inventarverzeichnis der Zweigbibliothek, sodass der Erwerbsvorgang durch das ehemalige Institut für Geschichte der Medizin eindeutig nachgewiesen werden konnte.

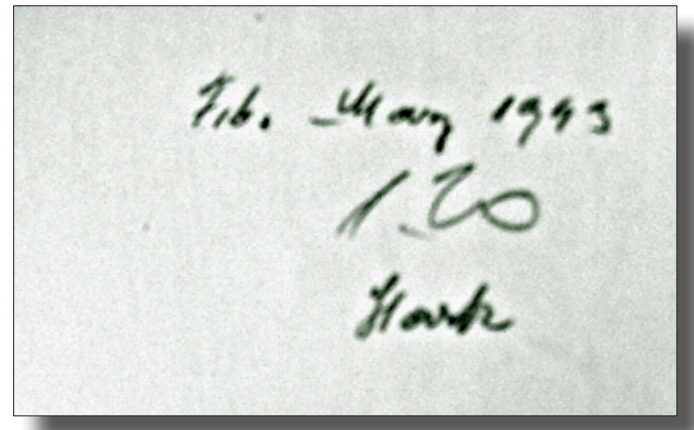

Abb. 5: Handschriftlicher Vermerk mit den Einträgen „Feb.-Marz 1943“, „1.20“, „Stark“

Am Titelblatt des Buches befindet sich ein Stempel mit der Aufschrift „Brüder Suschitzky, Spezialbuchhandlung und Antiquariat für sozialistische Literatur, Wien X“" als Hinweis auf früheren Besitzer des Buches.

Philipp Suschitzky, am 14. Februar 1876 in Wien geboren und später mit Olga Hirschler (1862-1942) verheiratet, war gelernter Buchhändler. Gemeinsam mit seinem Bruder Wilhelm (1877-1934) gründete er im Herbst 1901 in Wien X, Favoritenstraße 57, eine „Sortiments- und Antiquariatsbuchhandlung (mit angeschlossener Leihbibliothek)“, die 1911/12 durch 
die Gründung des „Anzengruber-Verlag Brüder Suschitzky“ erweitert wurde; benannt wurde der Verlag nach dem sozialkritischen Schriftsteller Ludwig Anzengruber. Mit beiden Unternehmungen verbanden die Brüder ihr kulturpolitisches Engagement im Umfeld der Sozialdemokratischen Partei Österreichs. In ihrem Verlagsprogramm wurden unter anderem pazifistisch-sozialistische Schriften, Bücher zur Frauenfrage, Freidenkertum, Sozial- und Sexualreform u.a. veröffentlicht. Bereits Ende der 1920er Jahre kam die Buchhandlung und deren Eigentümer aufgrund ihres Verlags- und Sortimentsprogrammes in den Brennpunkt der politischen-öffentlichen Auseinandersetzung und wurde vor allem in der Phase der Demokratiezerstörung der Jahre 1933/34 und danach zum Objekt beträchtlicher polizeilicher Repressalien (Hausdurchsuchungen, Buchkonfiskationen und anhängige Gerichtsverfahren). Nach dem Selbstmord von Wilhelm Suschitzky am 18. April 1934 trat seine Witwe, die am 19. November 1878 in Brünn geborene Adele Suschitzky, in die Firma ein.

1938 flohen Phillip und Olga Suschitzky nach Frankreich, von wo sie im September 1942 nach Auschwitz deportiert und hier ermordet wurden Adele Suschitzky flüchtete 1938 nach London, wo sie im Alter von 102 Jahren am 24. Mai 1980 gestorben ist.

Obwohl sich Johann Heger, ein ehemaliger Mitarbeiter der Firma, mittels Einschaltung diverser NS-Parteistellen um die „Arisierung“ des Unternehmens bemühte, kam es nach der Abweisung der „Arisierung“ im Oktober 1938 zur Schließung von Buchhandlung und Verlag, weil an einer Weiterführung dieses politisch brisanten Unternehmens seitens der Nationalsozialisten kein Interesse bestand. Das Bücherwarenlager wurde Anfang März 1939 in die Auktionshallen des Handelsgerichtes Wien überführt und dort veräußert. Das Buch, das durch den Abverkauf des Bücherwarenlagers durch das Handelsgericht Wien in den Antiquariatshandel gelangte, wurde vom Institut für Geschichte der Medizin im Februar/März 1943 vom Wiener Antiquariat „Karl Stark“ angekauft. Nach dem „Anschluss“ im März 1938 wurde das Antiquariat von Marianne Stern in Wien I., Karl Luegerplatz, von dessen früherem Mitarbeiter Karl Stark (*26.6.1886 Wien, + 13.5.1949 Wien) ,arisiert“. ${ }^{12}$

Wolf Suschitzky, der 1912 geborene Sohn von Wilhelm und Adele Suschitzky, absolvierte eine Foto-Ausbildung an der Höheren Graphischen Lehr- und Versuchsanstalt in Wien. Bereits 1934 emigrierte er nach London, wo er als Fotograf und Dokumentarfilmer erfolgreich tätig war und bis heute lebt. In jüngster Zeit thematisierten österreichische Zeitungen Leben und Werk von Wolf Suschitzky, darunter „Der Standard“13, „Die Presse“ ${ }^{\text {"14 }}$ und die „Wiener Zeitung“"15. Wolf Suschitzkys Bedeutung für den 
Film wurde in einer 2010 bei Synema Gesellschaft für Film und Medien erschienen Publikation gewürdigt. ${ }^{16}$

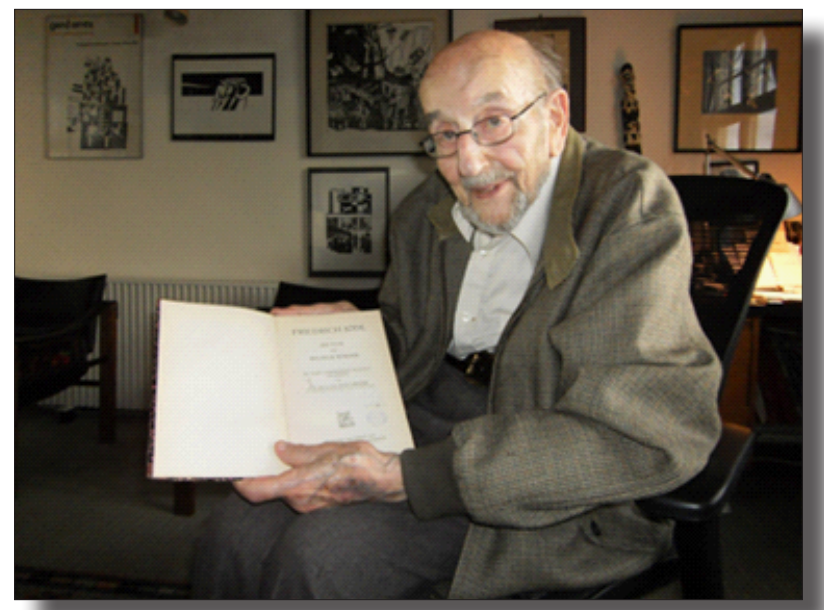

Abb. 6: Wolf Suschitzky mit dem restituierten Buch in seiner Wohnung in London (Foto: (c) Brigitte Mayr)

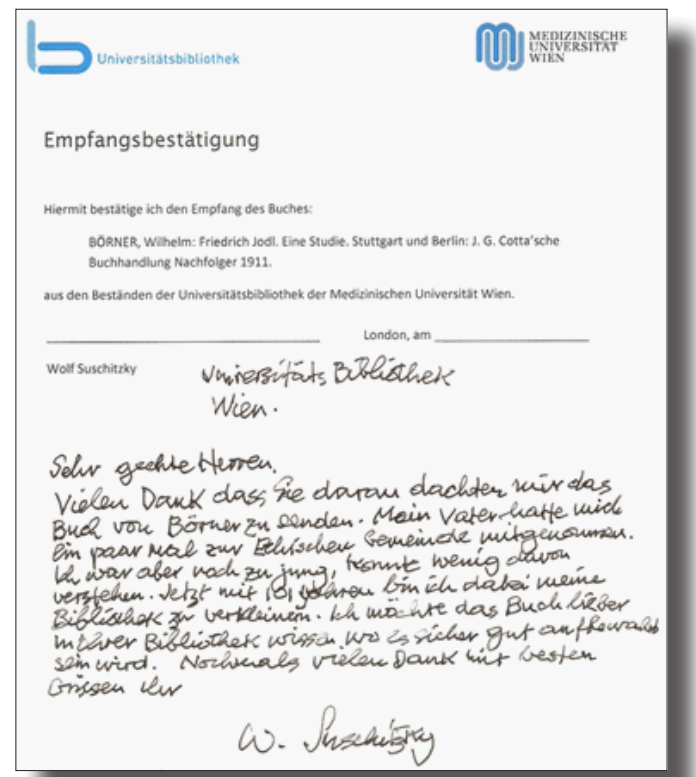

Abb. 7: Dankschreiben von Wolf Suschitzky mit der Überantwortung des Buches an die Universitätsbibliothek der Medizinischen Universität Wien 
Die Überreichung des Buches wurde im Auftrag der Medizinischen Universität Wien von Brigitte Mayr, einer Mitarbeiterin der Firma Synema Gesellschaft für Film und Medien, die bei Ausstellungs- und Publikationsprojekten sehr eng mit Wolf Suschitzky zusammenarbeitet, anlässlich eines Besuchs in London vorgenommen. Im Anschluss schenkte er, weil er seine Bibliothek verkleinern möchte, das Buch der Universitätsbibliothek der Medizinischen Universität Wien, „wo es sicher gut aufbewahrt sein wird“.

In ähnlicher Weise erfolgte durch die Universität Salzburg im November 2014 eine weitere Restitution an Wolf Suschitzky (Taschenwörterbuch der dänischen und deutschen Sprache, Langenscheidt-Verlag, 1907). ${ }^{17}$ Bereits im September 2013 war ein unrechtmäßig von der Universitätsbibliothek der Wirtschaftsuniversität Wien erworbenes Buch anlässlich eines WienBesuchs an Wolf Suschitzky restituiert worden (Schmidt, Heinrich: Was wir Ernst Haeckel verdanken, 1914). ${ }^{18}$

\section{Resümee}

Die beiden Restitutionsfälle, Antiquariat „Hans Peter Kraus“ bzw. Verlagsbuchhandlung „Anzengruber-Verlag, Brüder Suschitzky“, sind Bespiele für den an der Universitätsbibliothek der Medizinischen Universität Wien am häufigsten vertretenen Fall von Bücherraub. Aus den Lagerbeständen ,arisierter" und liquidierter Antiquariate und Buchhandlungen kamen zahlreiche geraubte Bücher in den Handel und in weiterer Folge an das Institut für Geschichte der Medizin und in dessen Rechtsnachfolge an die Medizinische Universität Wien bzw. deren Universitätsbibliothek. ${ }^{19}$ In beiden Fällen bestand glücklicherweise die Möglichkeit zur Kontaktaufnahme mit noch lebenden Kindern der Opfer des NS-Bücherraubes.

Nach den 2014 abgeschlossenen Restitutionsfällen „Hans Peter Kraus“ sowie „Anzengruber-Verlag, Brüder Suschitzky“ stehen an der Medizinischen Universität Wien für 2015 weitere ähnlich geartete Restitutionsfälle vor dem Abschluss. 
Mag. Bruno Bauer Universitätsbibliothek der Medizinischen Universität Wien Währinger Gürtel 18-20, A-1097 Wien E-Mail: bruno.bauer@meduniwien.ac.at Dr. Walter Mentzel
Zweigbibliothek für Geschichte der Medizin
an der Universitätsbibliothek der Medizinischen Universität Wien
Währinger Str. 25, A-1090 Wien
E-Mail: walter.mentzel@meduniwien.ac.at

1 Bruno Bauer und Walter Mentzel: Restitutionsfall Carl Julius Rothberger: erste Rückgabe von NS-Raubgut aus dem Bestand der Universitätsbibliothek durch die Medizinische Universität Wien. In: Mitteilungen der Vereinigung Österreichischer Bibliothekarinnen und Bibliothekare 63 (2010), H. 3/4, S. 101-107.

2 Bruno Bauer und Walter Mentzel: NS-Provenienzforschung an der Medizinischen Universität Wien 2011 und 2012. Restitution von Büchern der Bibliothek Sassenbach sowie den Privatbibliotheken von Raoul Fernand Jellinek-Merceds und Alfred Arnstein. In: Mitteilungen der Vereinigung Österreichischer Bibliothekarinnen und Bibliothekare 66 (2013), H. 3/4, S. 449-457.

3 Österreichisches Staatsarchiv (ÖSTA), Archiv der Republik (AdR), Bundesministerium für Finanzen (BMF), Vermögensverkehrsstelle (Wst), Vermögensanmeldung (VA), Zl. 50.691 Hans Peter Kraus.

4 Wiener Stadt- und Landesarchiv (WStLA), Volksgerichtsakt, A 1-VgVrStrafakt Vg 4 2.939/1945 Riedmann Friedrich Richard, sowie WStLA, Öffentliche Verwaltung (ÖVA) Alfred Wolf, Zeugeneinvernahme einer Mitarbeiterin von Alfred Wolf vom 21.5.1948.

5 Walter Mentzel: Wiener NS-Antiquariate und ihre Rolle im Bücherraub. Oder: Wie Antiquariate von der Judenverfolgung profitierten. Ein Forschungsbericht. In: Bruno Bauer, Christina Köstner-Pemsel und Markus Stumpf (Hrsg.): NS-Provenienzforschung an österreichischen Bibliotheken. Anspruch und Wirklichkeit (Schriften der Vereinigung Österreichischer Bibliothekarinnen und Bibliothekare, Band 10), Graz: Feldkirch: Neugebauer, 2011, S. 65-82. Online: http://eprints.rclis. org/17798/1/Schriften VOeB10 6582 MENTZEL Antiquariate.pdf

6 Walter Mentzel, Die „Antiquariats- und Exportbuchhandlung Alfred Wolf" - ehemals Hans Peter Kraus und Leo Weiser. Die Geschichte 
eines Raubunternehmens. In: Regine Dehnel (Hrsg.): NS-Raubgut in Museen, Bibliotheken und Archiven. Viertes Hannoversches Symposium (Zeitschrift für Bibliothekswese und Bibliographie, Sonderband 108), Frankfurt am Main 2012, S.441-454.

7 Hans Peter Kraus: A rare book saga: the autobiography of H. P. Kraus. New York:Putnam, 1978.

8 Hans Peter Kraus: Die Saga von den kostbaren Büchern. Ins Dt. übertr. von Lexa Katrin von Nostitz. Zürich: SV Internat., Schweizer Verl.-Haus, 1982.

9 Wolfgang Saxon: Hans Peter Kraus, 81, Book Dealer and Collector. In: The New York Times. 2. November 1988. Online: http://www.nytimes. com/1988/11/02/obituaries/hans-peter-kraus-81-book-dealer-andcollector.html

10 Hanni Kraus: In Memoriam Hans Peter Kraus 1907-1988. Hommage to a Bookman. Memorial service held on 29 November 1988 at the Pierpont Morgan Library. Speakers: T. Peter Kraus, Charles Pierce, William H. Scheide, Herbert Cahoon. Limited edition: one of the $400 \mathrm{cop}$ ies, printed at the press of A. Colish for the family H.P. Kraus.

11 Hans Peter und Hanni Kraus Collection of Sir Francis Drake. In: The Library of Congress/Rare Book \& Special Collections Reading Room (Website). Online: http://www.loc.gov/rr/rarebook/coll/kraus.html

12 Walter Mentzel: Wiener NS-Antiquariate und ihre Rolle im Bücherraub. Oder: Wie Antiquariate von der Judenverfolgung profitierten. Ein Forschungsbericht. In: Bruno Bauer, Christina Köstner-Pemsel und Markus Stumpf (Hrsg.): NS-Provenienzforschung an österreichischen Bibliotheken. Anspruch und Wirklichkeit (Schriften der Vereinigung Österreichischer Bibliothekarinnen und Bibliothekare, Band 10), Graz: Feldkirch: Neugebauer, 2011, S. 72-73. Online: http://eprints.rclis. org/17798/1/Schriften VOeB10 65 82 MENTZEL Antiquariate.pdf

13 Wolf Suschitzky: Chronist hinter der Kamera. In. derStandard.at (22.03.2007). Online: http://derstandard.at/2815233/Wolf-Suschitzky-Chronist-hinter-der-Kamera

14 Christoph Huber: WolfSuschitzky: Kameragenie aus Wien (14.03.2011). Online: http://diepresse.com/home/kultur/film/641839/Wolf-Suschitzky Kameragenie-aus-Wien

15 Das Kamera-Auge eines Jahrhunderts. Wolf Suschitzky zum Gespräch auf Radio Ö1 (12.12.2012). Online: http://www.wienerzeitung.at/nachrichten/kultur/medien/508635 Das-Kamera-Auge-eines-Jahrhunderts.html

16 Michael Omasta, Brigitte Mayr, Ursula Seeber: Wien: Synema - Ges. für Film und Medien, 2010. 
17 Universität Salzburg: Restitutionen 2014: Wiener Technischer Verlag Brüder Suschitzky. Online: http://www.uni-salzburg.at/index.php?id=55755

18 Wirtschaftsuniversität Wien: Provenienzforschung - Restitution. Online: http://www.wu.ac.at/ic/services/library/about/provenancen

19 Walter Mentzel und Bruno Bauer: Opfer des NS-Bücherraubes - 10 Fälle aus medizinischen Bibliotheken in Wien: Provenienzforschungsprojekt an der Universitätsbibliothek der Medizinischen Universität Wien. In: GMS Medizin Bibliothek Information 8 (2008), H. 3, Doc25. - Online: http:// www.egms.de/static/pdf/journals/mbi/2009-8/mbi000122.pdf

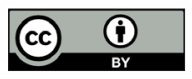

Dieses Werk ist lizenziert unter einer Creative-Commons-Lizenz Namensnennung 4.0 International 